\title{
Oscillator strengths between fine structure levels of Fe xxIII
}

\author{
J. A. Tully ${ }^{1}$ and M. C. Chidichimo ${ }^{2}$ \\ 1 Observatoire de la Côte d'Azur, CNRS Laboratoire Cassini, Bd. de l'Observatoire, BP 4229, \\ 06304 Nice Cedex 4, France \\ 2 Department of Applied Mathematics, University of Waterloo, Waterloo, Ontario N2L 3G1, Canada
}

Received 18 July 2000 / Accepted 4 October 2000

\begin{abstract}
We tabulate theoretical line strengths, $f$-values and transition energies for the beryllium-like ion Fe XXIII. Transitions are between levels $2 l_{1} 2 l_{2} S^{\prime} L^{\prime} J^{\prime}$ and $2 l_{3} n l_{4} S L J$ with $n=2,3$, 4 . The calculation uses the well known configuration interaction program CIV3 in which relativistic effects are allowed for by means of the Breit-Pauli approximation. We give a detailed comparison of our oscillator strengths with those which Chen \& Ong (1998) obtained using the relativistic Dirac code GRASP2.
\end{abstract}

Key words. Atomic data

\section{Introduction}

Numerous papers dealing with the radiative properties of Fe XXIII have appeared over the past few years and this is proof of the interest that this highly ionised Be-like ion continues to arouse. As the last millenium draws to a close two veterans in the field of atomic structure calculations have presented energy levels and oscillator strengths for beryllium-like ions including Fe XXIII (Kingston \& Hibbert 2000).

Those wishing to compute collision strengths for electron excitation of an ion need wavefunctions for the ground and several excited states. As part of the IRON Project, Chidichimo et al. (1999) - hereafter CZTB calculated analytic radial orbitals for Fe XXIII with the program CIV3 (Hibbert 1975) and used these to produce oscillator strengths for transitions within the ground complex: $2 l_{1} 2 l_{2} \rightarrow 2 l_{3} 2 l_{4}$. These were the allowed transitions for which they computed collision strengths. Here we extend their work by calculating line and oscillator strengths for many more transitions and compare our results with those drawn from the work of several other authors. Of special interest is the detailed comparison we make with the results of Chen \& Ong (1998) - hereafter CO - who used the unpublished code called GRASP2, written by F.A. Parpia, I.P. Grant and C. Froese-Fischer. GRASP2 is based on multiconfiguration Dirac-Fock Theory and so makes allowance for relativistic effects in a fuller manner

Send offprint requests to: J. A. Tully

* Tables 1 to 17 are only available in electronic form at http://www.edpsciences.org than we do, our calculation being based on the Breit-Pauli approximation (Hibbert et al. 1991).

\section{Atomic orbitals and configurations}

Our atomic orbitals $P_{n l}(r)$ are Slater type and have the following analytic form

$P_{n l}(r)=\sum_{j=1}^{k} c_{j n l} \frac{\left(2 \zeta_{j n l}\right)^{I_{j n l}+\frac{1}{2}}}{\left[\left(2 I_{j n l}\right) !\right]^{\frac{1}{2}}} r^{I_{j n l}} \exp \left(-\zeta_{j n l} r\right)$.

We take $P_{1 \mathrm{~s}}$ and $P_{2 \mathrm{~s}}$ from Clementi \& Roetti (1974) and calculate $P_{3 \mathrm{~s}}, P_{4 \mathrm{~s}}, P_{2 \mathrm{p}}, P_{3 \mathrm{p}}, P_{4 \mathrm{p}}, P_{3 \mathrm{~d}}, P_{4 \mathrm{~d}}, P_{4 \mathrm{f}}$ using CIV3. Values of the orbital parameters (i.e. coefficients and exponents) are given in Table 1 . Some of the values differ from those found by CZTB. The explanation is simply that in the present work we allowed the number of iterations in the optimisation branch of CIV3 to exceed five, the value Hibbert (1975) recommends. Only the parameters of the $3 \mathrm{~s}, 4 \mathrm{~s}, 4 \mathrm{p}$ orbitals are affected to any noticeable extent, but the resulting radial functions are numerically similar to the ones in CZTB.

We use configurations $1 \mathrm{~s}^{2} n_{a} l_{a} n_{b} l_{b}$ in which $n_{a}$ and $n_{b}$ can take the values $2,3,4$. This leads to 295 fine structure states of which 146 (149) have odd (even) parity. Details of these single configuration states are given in Tables 2 and 3 . CIV3 calculates the mixing amongst states having the same parity and $J$ value. Each final state is a mixture of configurations and usually, but not always, is dominated by one of them which serves as a convenient label. For further details see CZTB. 
Tables 4 through 13 contain length and velocity line strengths $S_{\mathrm{l}}$ and $S_{\mathrm{v}}$ in atomic units; length and velocity absorption oscillator strengths $f_{\mathrm{l}}$ and $f_{\mathrm{v}}$; and calculated transition energies $E_{i j}$ in rydberg $(\mathrm{Ry})$ units.

\section{Comparison with the work of others}

Table 14 is a detailed comparison of our $f$-values and those of $\mathrm{CO}$ who made use of an entirely different method from us. Furthermore, CO used 78 fine structure states compared to our 295 since they restricted the quantum numbers $n_{a}$ and $n_{b}$ to 2 and 3. For 28 of the 243 transitions which are listed in Table 14, our $f$-values and those of CO differ by less than 0.5 per cent. For 144 transitions the differences are between $0.5 \%$ and $5 \%$; for 38 transitions between 5\% and 10\%; for 19 transitions between $10 \%$ and $25 \%$. And finally for 13 transitions, the $f$-values differ by more than $25 \%$ but less than $72 \%$; however, 12 of these $f$-values are quite small. The exception is the transition $2 \rightarrow 34$, namely $2 \mathrm{~s} 2 \mathrm{p}^{3} \mathrm{P}_{0}^{\mathrm{o}} \rightarrow 2 \mathrm{p} 3 \mathrm{p}^{3} \mathrm{~S}_{1}$, where $f(\mathrm{CO})=0.001846$ and $f(\mathrm{TC})=0.001169$.

Table 15 compares the energy levels we obtain from a 78 state calculation using CIV3 with the energies from CZTB and CO. Note that levels with indexes 13 and 14 are almost degenerate in energy; CO find they are ordered differently from CZTB. In other words, their level 13 (14) is level 14 (13) in CZTB. It is interesting to note that when we use 295 states the order of these two levels agrees with that given by $\mathrm{CO}$.

In Table 16 we give $f$-values for transitions within the ground complex and results to compare with are taken from several sources: Cheng et al. (1979), Nussbaumer \& Storey (1979), Bhatia \& Mason (1981, 1986), Safronova et al. (1992), Zhang \& Sampson (1992). It is possible that Safronova et al. (1992) made a slip when tabulating their results for the transitions $2 \mathrm{~s}^{2}{ }^{1} \mathrm{~S}_{0} \rightarrow 2 \mathrm{~s} 2 \mathrm{p}^{3} \mathrm{P}_{1}^{\mathrm{o}},{ }^{1} \mathrm{P}_{1}^{\mathrm{o}}$ since the numbers they give differ by about a factor of 3 from those obtained by everyone else. Table 17 compares our line strengths for transitions $2 l_{1} 2 l_{2} \rightarrow 2 l_{3} n l_{4}, n=2,3$ with those given by Sampson et al. $(1980,1984)$.

\section{A final comment}

The present investigation originated as an offshoot to CZTB. Claudio Mendoza and co-workers are calculating radiative data for Fe XXIII with the R-matrix codes and their results will appear in due course in the "Atomic data from the IRON Project" series.

Acknowledgements. Alan Hibbert kindly added an appropriate written statement to his program so making it easier to extract large numbers of line strengths and $f$-values from CIV3.

\section{References}

Bhatia, A. K., \& Mason, H. E. 1981, A\&A, 103, 324

Bhatia, A. K., \& Mason, H. E. 1986, A\&A, 155, 413

Chen Guo-xin, \& Ong, P. P. 1998, Phys. Rev. A, 58, 1070

Cheng, K. T., Kim, Y.-K., \& Desclaux, J. P. 1979, At. Data Nucl. Data Tables, 24, 111

Chidichimo, M. C., Zeman, V., Tully, J. A., \& Berrington, K. A. 1999, A\&AS, 137, 175 (Errata: 2000, A\&AS, 143, 541)

Clementi, E., \& Roetti, C. 1974, At. Data Nucl. Data Tables, 14,177

Hibbert, A. 1975, Comput. Phys. Commun, 9, 141

Hibbert, A., Glass, R., \& Froese Fischer, C. 1991, Comput. Phys. Commun, 64, 455

Kingston, A. E., \& Hibbert, A. 2000, J. Phys. B, 33, 693

Nussbaumer, H., \& Storey, P. J. 1979, J. Phys. B, 12, 1647

Safronova, U. I., Shlyapzeva, A. S., Vainshtein, L. A., Kato, T., \& Masai K. 1992, Phys. Scr, 46, 409

Sampson, D. H., Clark, R. E. H., \& Golden, L. B. 1980, ApJS, 44, 193

Sampson, D. H., Goett, S. J., \& Clark, R. E. H. 1984, At. Data Nucl. Data Tables, 30, 125

Zhang, H. L., \& Sampson, D. H. 1992, At. Data Nucl. Data Tables, 52, 143 